\title{
Harnessing GPU Tensor Cores for Fast FP16 Arithmetic to Speed up Mixed-Precision Iterative Refinement Solvers
}

DOI:

10.1109/SC.2018.00050

\section{Document Version}

Accepted author manuscript

Link to publication record in Manchester Research Explorer

\section{Citation for published version (APA):}

Haidar, A., Tomov, S., Dongarra, J., \& Higham, N. (2018). Harnessing GPU Tensor Cores for Fast FP16 Arithmetic to Speed up Mixed-Precision Iterative Refinement Solvers. In SC '18: Proceedings of the International Conference for High Performance Computing, Networking, Storage, and Analysis (Vol. 0, pp. 1-11). [47] IEEE. https://doi.org/10.1109/SC.2018.00050

\section{Published in:}

SC '18: Proceedings of the International Conference for High Performance Computing, Networking, Storage, and Analysis

\section{Citing this paper}

Please note that where the full-text provided on Manchester Research Explorer is the Author Accepted Manuscript or Proof version this may differ from the final Published version. If citing, it is advised that you check and use the publisher's definitive version.

\section{General rights}

Copyright and moral rights for the publications made accessible in the Research Explorer are retained by the authors and/or other copyright owners and it is a condition of accessing publications that users recognise and abide by the legal requirements associated with these rights.

\section{Takedown policy}

If you believe that this document breaches copyright please refer to the University of Manchester's Takedown Procedures [http://man.ac.uk/04Y6Bo] or contact uml.scholarlycommunications@manchester.ac.uk providing relevant details, so we can investigate your claim.

\section{OPEN ACCESS}




\title{
Harnessing GPU Tensor Cores for Fast FP16 Arithmetic to Speed up Mixed-Precision Iterative Refinement Solvers
}

\author{
Azzam Haidar*, Stanimire Tomov*, Jack Dongarra* ${ }^{* \dagger}$ Nicholas J. Higham $\S$ \\ \{haidar|tomov|dongarra\}@ icl.utk.edu, \\ *Innovative Computing Laboratory (ICL), University of Tennessee, Knoxville, USA \\ $\dagger$ Oak Ridge National Laboratory, USA \\ \#University of Manchester, UK \\ ${ }^{\S}$ School of Mathematics, University of Manchester, UK
}

\begin{abstract}
Low-precision floating-point arithmetic is a powerful tool for accelerating scientific computing applications, especially those in artificial intelligence. Here, we present an investigation showing that other high-performance computing (HPC) applications can also harness this power. Specifically, we use the general HPC problem, $A x=b$, where $A$ is a large dense matrix, and a double precision (FP64) solution is needed for accuracy. Our approach is based on mixed-precision (FP16 $\rightarrow$ FP64) iterative refinement, and we generalize and extend prior advances into a framework, for which we develop architecture-specific algorithms and highly tuned implementations. These new methods show how using half-precision Tensor Cores (FP16-TC) for the arithmetic can provide up to $4 \times$ speedup. This is due to the performance boost that the FP16-TC provide as well as to the improved accuracy over the classical FP16 arithmetic that is obtained because the GEMM accumulation occurs in FP32 arithmetic.
\end{abstract}

Index Terms-FP16 Arithmetic, Half Precision, Mixed Precision Solvers, Iterative Refinement Computation, GPU Computing, Linear Algebra

\section{INTRODUCTION}

To take advantage of new processor designs, algorithms must also be redesigned. This is especially true and challenging in the area of dense linear algebra, where many algorithms are expected to run at close to the machine's peak performance. For example, LINPACK was redesigned to move away from using vector algorithms that were useful on the vector machines of the 1970s, leading to the new Linear Algebra PACKage (LAPACK) that uses blocked algorithms on cache-based processors. LAPACK itself had to be redesigned for multi-core and heterogeneous many-core architectures, which resulted in the Matrix Algebra on GPU and Multicore Architectures (MAGMA) library [15], [26].

This paper discusses the redesign of a mixed-precision iterative refinement technique to harness the fast FP16-TC arithmetic available in the latest NVIDIA GPUs. Modern architectures are trending toward multiple floating-point arithmetic precisions being supported in the hardware, and lower precisions are often much faster than higher precisions. For example, single-precision, 32-bit floating-point arithmetic (FP 32) is usually twice as fast as double-precision, 64-bit floating-point arithmetic (FP64). Recently, various machine learning and artificial intelligence neural network applications increased the need for FP 16 arithmetic (see Figure 1), and vendors started to accelerate it in hardware. Currently, the NVIDIA V100 TCs can execute FP16 at up to 112 teraFLOP/s ( 85 teraFLOP/s when the matrix $A$ and $B$ are in FP32) - vs. 7 teraFLOP/s for FP 64 and 14 teraFLOP/s for FP 32 on a V100 through PCIe. Developing algorithms to use this hardware efficiently will be highly beneficial in high-performance computing (HPC).

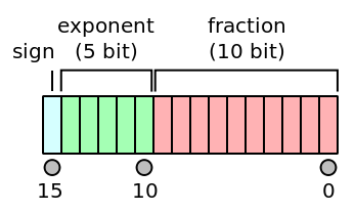

Fig. 1. IEEE 754 FP 16 format. This representation has 3.311 decimal digits of accuracy and a maximum representable value of 65,504 .

Mixed-precision iterative refinement is used to accelerate $A x=b$ solvers, where $A$ is a dense matrix. The main idea is to compute the $\mathrm{LU}$ factorization of $A$ in low precision and use the factorization in a refinement loop, with the residual possibly computed in higher precision. These methods have been studied in the past, as discussed in Section [I] A persistent challenge has been to redesign the techniques for new architectures and to develop highly tuned implementations that resolve computational issues like inefficient parallelization, scaling, and use of mixed-precision calculations. A lot of the theoretical work with numerical experiments on small problems has been restricted on MATLAB or reference implementations, which are prone to overlook computational issues when achieving acceleration using highly tuned standard solvers. To address this problem on GPU Tensor Cores, we leverage building blocks from the MAGMA library, which provides state-of-the-art, high-performance algorithms such as LU factorization-including a set of highly tuned mixedprecision iterative refinement algorithms for FP $32 \rightarrow$ FP 64 arithmetic [27]. 


\section{RELATED WORK}

Iterative refinement is a well-established technique that dates back to Wilkinson in the 1940s. The idea is to improve the computed solution of a linear system by solving a correction equation and adding the correction to the original solution; see Wilkinson [28], Moler [19], Stewart [25], Demmel [7]) and, for a comprehensive treatment, Higham [14, Chap. 12]. In iterative refinement, the three tasks (original solve/factorization, residual computation, and correction equation solve) can be done in the same precision (fixed precision) or in different precisions (mixed precision). Fixed precision iterative refinement was analyzed by Skeel [24] for an LU solver and extended by Higham [12], [13] for a general solver. In the 2000s, motivated by processors equipped with FP 32 speed $2 \times$ that of $\operatorname{FP} 64$, mixed precision iterative refinementwith the LU factorization done in FP 32 and everything else done in FP 64-was explored in [3], [17].

Replacing the direct triangular solves of the correction equation with an iterative method, as suggested in [4] in a mixed precision context, leads to "nesting" of two iterative methods, which in general are called "inner-outer" iterations, the latter having been studied both theoretically and computationally [9], [21], [23], including in mixed-precision computation scenarios [2]. Recently, Carson and Higham [4], [5] analyzed the convergence property of a three precision iterative refinement scheme (factorization precision, working precision, residual precision) and concluded that if the condition number of $A$ is not too large, $\kappa_{\infty}(A)=\|A\|_{\infty}\left\|A^{-1}\right\|_{\infty}<10^{4}$, then using FP1 6 for the $O\left(n^{3}\right)$ portion (the LU factorization) and (FP 32, FP 64) or (FP 64, FP 128) as the (working, residual) precision for the $O\left(n^{2}\right)$ portion (refinement loop), one can expect to achieve forward error and backward error on the order of $10^{-8}$ and $10^{-16}$ respectively. We note that, if $\hat{x}$ is the solution of $A x=b$ the forward error is defined by $\|\hat{x}-x\|_{\infty} /\|x\|_{\infty}$ and the backward error is defined by $\|r\|_{2} /\|A\|_{2}\|\hat{x}\|_{2}$ where $r=b-A \hat{x}$. The same study also showed that when using the generalized minimal residual (GMRES) method preconditioned by the FP16 LU factorization as the refinement procedure, the constraint on the condition number can be relaxed to be $\kappa_{\infty}(A)<10^{8}$ when the (working, residual) precision is (FP 32 , FP 64 ) and to $10^{12}$ when the (working, residual) precision is (FP64, FP128).

An investigation of similar iterative refinement methods on earlier generations of GPUs can be found in [11]. With the announcement of NVIDIA's V100 Tensor Cores. which improve numerical precision and speed for $\mathrm{FP} 16$, it is our intention to comprehensively investigate how the V100 opens a new world of opportunities in matrix computations.

\section{CONTRIBUTIONS}

Compared to our previous work in [11], the primary contribution of this paper is to propose and implement a high-performance framework for the mixed-precision iterative refinement solvers that makes use for the first time of GPU Tensor Core-accelerated FP16-TC. To this end, we will:
- introduce a new class of multi-precision dense matrix factorization algorithms; and here we mean that the factorization itself is implemented in multi-precision despite the fact the iterative refinement is mixed precision.

- develop a framework for exploiting GPU TCs in mixed-precision (FP16-FP32/FP64) iterative refinement solvers and describe the path to develop highperformance, Tensor Cores-enabled dense linear algebra building blocks kernels that can be used to exploit the FP16-TC in HPC applications;

- present a study on algorithmic variants of IR techniques;

- include performance model study that allow users/developers to understand the effect of the number of iterations and to predict performance gain;

- illustrate that a number of problems can be accelerated up to $4 \times$ through the mixed-precision solvers using fast FP16-TC, 3× using the basic FP16 mixed-precision solver, or $2 \times$ using the FP 32 arithmetic;

- provide an analysis of the numerical behavior of the proposed mixed-precision, TC-accelerated solvers on different types of matrices; and

- quantify - in practice-the performance and limitations of this approach on V100 GPUs using TC;

- we also provide experiments on dense and sparse matrices arising from real applications (see Table III);

The developments will be released through the open-source MAGMA library [15] to make these experiments independently reproducible and to allow the scientific community build and study different type of research on the top of this work. We also would like to point readers who are interested in energy efficiency and power measurement to our work presented in [10] and which investigate the energy gain that can be brought by the iterative refinement techniques from a power consumption point of view.

\section{Methods}

We consider two methods to extract higher precision solutions from low-precision factorizations. The first method is standard iterative refinement (IR) as implemented in LAPACK, and the second method is iterative refinement using preconditioned GMRES for solving the correction equation, which we denote by IRGM. The IR method is a wellestablished technique, while IRGM is more recent and holds more promise for exploiting low precision in factorizations.

\section{A. Background}

Below we expound on the IR and IRGM methods.

1) The $L U$ factorization: An LU factorization represents $A$ as the product of a lower triangular matrix $L$ and and an upper triangular matrix $U$, so that solving $A x=b$ reduces to solving two triangular systems:

$$
A x=b \Rightarrow L U x=b: \text { solve } L y=b \text { then solve } U x=y .
$$

Algorithmically, as illustrated in Figure 2, the LU factorization can be viewed as a sequence of steps with two distinct phases per step: 1) a panel factorization that affects the data depicted 
by the orange portion of Figure 2, and 2) a trailing matrix update that updates data represented by the magenta and green colors in Figure 2. From a software point of view, we know that PanelFactorize is a memory-bound step performed through the Xget $f 2$ routine and occupies a small portion of the total time, while TrailingMatrixUpdate is compute-bound and is performed using the BLAS-3 routines (Basic Linear Algebra Subprograms) Xtrmm and Xgemm and occupies the greatest portion of the time spent in the factorization. Thus one might expect the performance of the LU factorization to be asymptotically similar to the BLAS-3 Xgemm routine.

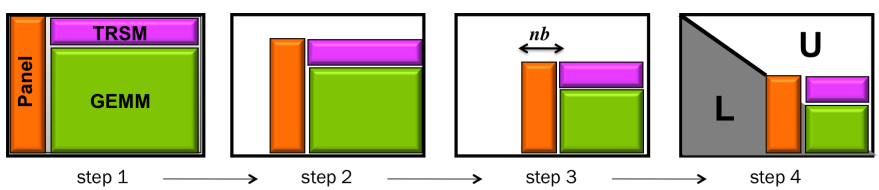

Fig. 2. Two-phase implementation of a one-sided factorization.

2) Iterative Refinement: The IR technique improves the accuracy of a computed solution, $\hat{x}$, for a linear system of equations, $A x=b$. Here, we let the initial solution be $x_{0}=0$, and the iterative refinement is a series of iterations:

1) Residual: Compute the residual $r=b-A x_{i}$.

2) Correction: Solve $A c=r$ (e.g., using an initial LU factorization).

3) Update: Correct the current solution $x_{i+1}=x_{i}+c$.

If all three steps can be computed exactly, then the IR algorithm completes in one iteration. However, in floatingpoint arithmetic the above iterations must be repeated.

IR is usually carried out with LU factorization with partial pivoting. In this context, the correction equation is solved using the LU factors. Denote by $\boldsymbol{u}$ the precision in which steps 1 and 3 are carried out. Step 2 is performed in the precision $\boldsymbol{u}_{f}$ which is the precision of the LU factorization, because it uses the "L" and "U" factors to solve the correction equation $A c=r$ and then it casts the solution " $c$ " to the working precision $\boldsymbol{u}$. If the LU factorization is also computed in the precision $\boldsymbol{u}$, the method is called fixed-precision IR, otherwise if the $\mathrm{LU}$ factorization is performed at lower precision $\boldsymbol{u}_{f}$, it is called mixed-precision IR. Fixed-precision IR can be used to improve the backward error of an LU factorization without a strong stabilizing pivoting strategy [1], [8], [18], [24]. On the other hand, mixed-precision IR also improves the forward error to the working precision-if the condition number of $A$ is not too large: $\boldsymbol{u}_{f} \kappa_{\infty}(A) \leq 1$.

The economics of mixed-precision IR using low-precision LU factorization compared with directly solving with a higher precision LU factorization depend on the relative speed of low-precision arithmetic and on the cost of the refinement process. It is therefore a function of the executing hardware, the IR configuration, and also of the properties of the matrix $A$ (notably, its condition number). In the specific case of the V100 GPU, the practical speed of FP16-TC is about $12 \times$ faster than EP 64 for square $\mathrm{Xgemm}$ and is about $6 \times$ faster for the rank-k update Xgemm that is used by the LU factorization (see Figure 3a) which pushes the balance in favor of mixedprecision IR.

3) Iterative Refinement with Preconditioned GMRES: GMRES [22] is a popular Krylov subspace iteration for solving a general linear system of equations. Following Carson and Higham [4] we will consider another variant of iterative refinement by using preconditioned GMRES to solve the correction equation $A c=r$ in step 2 of the classical IR algorithm described above. GMRES will be preconditioned by the low precision LU factors. The idea is that the GMRES solver will provide a better and more stable solution to $A c=r$ than the basic triangular solve, which is directly affected by the quality of the low precision LU factors. Using GMRES we can still guarantee that the solution of the correction equation $A c=r$ has residual at the level of the convergence tolerance requested by the algorithm. The convergence tolerance is chosen of the order of the unit roundoff of the low precision arithmetic used during the factorization (e.g., we use $10^{-4}$ or $10^{-8}$ for when the LU is in FP16 or FP 32 respectively). Since this paper focuses on practical usage and possible performance gains rather than error analysis, we point the reader to [4], [5] for detailed error analysis of the IR and IRGM techniques.

We describe both the IR and IRGM methods in Algorithm 1 in a unified framework, where the difference between them is in the correction equation solver ( $\mathrm{LU}$ or preconditioned GMRES).

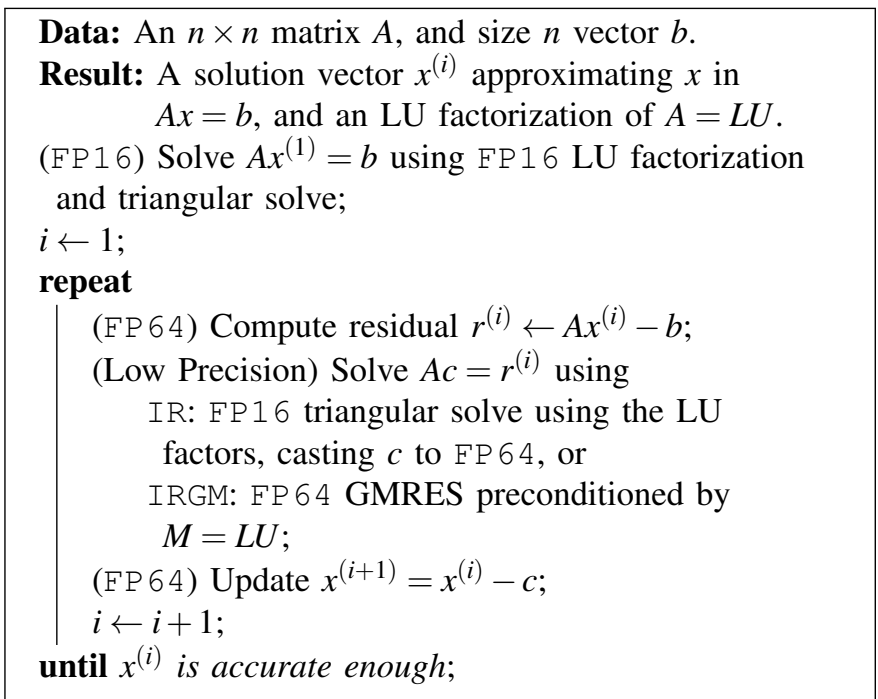

Algorithm 1: IR: mixed-precision iterative refinement using triangular solve. IRGM: mixed-precision iterative refinement with GMRES to solve correction equation.

\section{B. Algorithmic Advancements}

A recent study by Carson and Higham [4] provides an analysis of using three precisions for the IR iterations, which we can take as FP 32 for the working precision, FP1 6 for the LU factorization, and EP 64 for the residual computation (Table I). For a matrix with a condition number of $\kappa_{\infty}(A)<10^{4}$, IR converges to FP32 accuracy. However, when the correction equation is solved by GMRES preconditioned by the LU 
TABLE I

IEEE PRECISIONS AND NUMERICAL PROPERTIES.

\begin{tabular}{lll} 
Type & Range & Unit Roundoff \\
\hline FP16 & $10^{ \pm 5}$ & $5 \times 10^{-4}$ \\
FP32 & $10^{ \pm 38}$ & $6 \times 10^{-8}$ \\
FP64 & $10^{ \pm 308}$ & $1 \times 10^{-16}$ \\
\hline
\end{tabular}

factors and the matrix-vector products are computed in FP 64 , the restriction on the condition number can be relaxed to $\kappa_{\infty}(A)<10^{8}$. However, it remains unclear how fast GMRES converges. Inspired by the analysis of the aforementioned study and the performance potential of FP16 on the NVIDIA V100 GPUs, we have developed a practical implementation of a similar IR variant with GMRES denoted by IRGM in the MAGMA library [15]. The implementation is different from [4] in that:

- 1) we only use two precisions-FP16 for the LU factorization $O\left(n^{3}\right)$ work and $\operatorname{FP} 64$ for the high precision for everything else; This decision is driven by the work done by [3], [17] which studied and showed the feasibility of the two precisions iterative schema and by the complexity required to implement three precision algorithm in particular the quad precision.

- 2) we propose a multi-precision factorization algorithm. Our LU algorithm uses both FP16 and FP 32 precision during its progress. We only use FP16 matrix multiplication (hgemm) in the LU factorization, while everything else is in FP32-effectively making our LU multi-precision. This decision is driven by the idea to compute the most numerically sensitive portions (e.g., the panel factorization and the trsm) of the algorithm in higher precision to avoid underflow and overflow that can easily occur when operating in full FP16.

\section{Half-Precision LU using V100 Tensor Cores}

Driven primarily by the need for training in deep learning, the latest offering from NVIDIA - the Tesla V100 GPU based on the Volta architecture-provides specific programmable matrix multiply-accumulate units that are said to deliver a theoretical peak performance of 110 teraFLOP/s in FP16-TC. The V100 has 8 Tensor Cores per streaming processor for a total of 640 Tensor Cores. A Tensor Core can compute $D=A * B+C$ per clock cycle, where all matrices are $4 \times 4$ in size (i.e., 64 floating point FMA mixed-precision operations per clock cycle). $A$ and $B$ must be in FP16, but $C$ and $D$ can be in either FP16 or FP32. The multiplication occurs in FP16 and is accumulated in FP 32 with other products. In our experiment, FP16-TC denotes the use of the Tensor Core EP16 routine. We expect significant improvements in numerical behavior over the basic FP16 arithmetic, where all calculations are rounded and accumulated in FP16. The Tensor Core caters primarily to deep learning applications, where lower precision is tolerable, and is not straightforward for use in more numerically demanding applications (e.g., numerical simulations and linear solvers in particular). Since TC provides a large speedup for matrix-multiplication, it can be expected to accelerate FP16 dense matrix factorizations, which are rich in matrix multiplication operations. The challenge lies in how to use the low-precision factorization results to achieve FP32/FP 64 level accuracy effectively and efficiently.

\section{Convergence Consideration}

This subsection discusses the convergence rate of the IR and IRGM methods in Algorithm 1 Analysis for mixed precision iterative refinement can be found in [4], [5]. It yields the sufficient condition $\kappa_{\infty}(A)<10^{4}$ for linear convergence of IR. It would provide the sufficient condition for convergence $\kappa_{\infty}(A)<10^{12}$ for IRGM if quadruple precision were used in computing the residuals and applying the preconditioned matrix. However, it can be shown that our implementation of IRGM guarantees convergence as long as $\kappa_{\infty}\left(U^{-1} L^{-1} A\right) \kappa_{\infty}(A)<10^{16}$, which holds if $\kappa_{\infty}(A)<10^{8}$ (the rounding error analysis leading to these conclusions will be reported elsewhere).

For IRGM, the number of GMRES iterations required per refinement step is difficult to predict, and the low accuracy FP16 preconditioner means we have little knowledge about the preconditioned matrix. In general, for a normal matrix, $A$, GMRES converges more slowly as the condition number of $A$ increases. For a non-normal matrix, the convergence rate cannot be predicted by the condition number alone. In practice, the convergence rate depends on the matrix type, the condition number, and the matrix size. Therefore, in the next section we study our two proposed algorithms by trying matrices with different spectrum, sizes, and types.

\section{Analysis And Expected Performance}

The primary motivation for using FP16 arithmetic is its unprecedented high performance compared with higher precisions. This performance is quantified for the V100 GPU in Figure 3. The PCIe V100 has a practical peak of 6.8 teraFLOP/s in FP 64, 14 teraFLOP/s in FP 32, 28 teraFLOP/s in FP16, and a remarkable 85 teraFLOP/s in FP16-TC (Tensor Cores). The performance of the $\mathrm{LU}$ factorization relies mostly on the performance of the Schur update (or rank-k Xgemm update), which is a tall-skinny matrix multiplication that occurs during each step of the LU algorithm. For that, to understand/model how the LU factorization could benefit from the FP16 arithmetic, we first study the performance of the Xgemm routine. This is shown in Figure $3 \mathrm{a}$ for the four available precisions (FP 64, FP 32, FP16, and FP16-TC). We consider FP16-TC as a separate precision, since it consists of a mixed-precision Xgemm, where the multiplication is performed in FP16, while the accumulation is in FP32. Thus, FP16-TC is more accurate than a homogeneous FP16 computation. We also note that, in addition to being more accurate, the FP16-TC is also much faster due to the use of Tensor Cores.

As shown in Figure 3a, the FP16-TC hgemm-TC operating on square matrices is about $12 \times$ faster than its FP $64 \mathrm{dgemm}$ counterpart. Furthermore, and as expected, the FP16 hgemm 
is $2 \times$ faster than the FP 32 sgemm and about $4 \times$ faster than the FP64 dgemm. Figure 3 a also depicts the performance of the Xgemm for a rank-k update (dashed lines). This is the type of operation needed by the LU and thus it gives us an indicator of the performance ceiling/bound of any LU implementation. The rank-k update hgemm-TC is slower than the square hgemm-TC due to the fact that the time to read the data of $A$ and $B$ is comparable to the computational time for the rank-k update in half precision TC while it is a lot smaller for the square case or for higher precision, but it still carries an attractive speedup compared to the dgemm. The rank-k hgemm-TC achieves about 35 teraFLOP/s, compared to about 25 teraFLOP/s for the rank-k hgemm, 13 teraFLOP/s for the rank-k sgemm, and around 6 teraFLOP/s for the rank-k dgemm.

We also developed a multi-precision LU factorization in FP16, where we performed the numerically sensitive portion of the code (e.g., the panel factorization and the trsm) in FP 32-only the GEMMs are in FP16 or FP16-TC, and any value that exceeds the FP1 6 range is rounded to the nearest finite floating-point number. The idea here is to provide a better stable LU factorization than the fully FP1 6 implementation without loss of performance. Figure $3 \mathrm{~b}$ shows the performance for the four precisions. As expected, our LU implementation follows roughly the same trend as the Xgemm kernel for large $n$, which proves that our implementation is very well optimized and is able to attain the theoretical upper bound. Our hgetrf and hgetrf-TC solvers achieve a speedup from $4 \times$ to $5 \times$ over dgetrf respectively and a $2 \times$ speedup over sget $\mathrm{f}$.

This section is dedicated to the theoretical performance analysis of the mixed precision (MP) algorithms for linear solvers. The idea is to understand and predict when iterative refinement techniques can be used in a beneficial fashion. From a performance point of view, an algorithm is beneficial when it reaches the solution in a time faster than the reference one (which is the FP64 dgesv routine in our case). The iterative refinement solvers consist of an LU factorization in low precision $\varepsilon_{F P X X}<\varepsilon_{F P 64}$ followed by an iterative loop based on either classical IR or GMRES (as described above) to improve the solution to $\varepsilon_{F P 64}$. Thus, let us define

$$
\begin{gathered}
\text { time for FP64 }=\frac{2 n^{3}}{3 P_{\text {dgetrf }}}+\frac{2 n^{2}}{P_{d t r s v}} \\
\text { time for } \mathrm{MP}=\frac{2 n^{3}}{3 P_{X g e t r f}}+k\left(\frac{2 n^{2}}{P_{\text {dgemv }}}+\frac{2 n^{2}}{P_{X \text { trsv }}}+\xi\right)
\end{gathered}
$$

where $P$ denotes the performance of the corresponding routine; $k$ denotes the number of iterations required by the MP solver to achieve the double precision solutions, including the inner GMRES iterations in the case of the IRGM solver; and $\xi$ refers to the other work required by the iterative refinement such as norm computation, residual calculation, pivoting, synchronizations. In our experiments we found that $\xi$ is negligible compared with the cost of the dgemv and the Xtrsv.

Based on the LU performance results provided in Figure $3 b$ and on the benchmark of the dgemv and Xtrsv routine, we illustrate in Figure $\mathrm{V}$ the expected speedup of our three $\mathrm{MP}$ routines (e.g., dhgesv-TC, dhgesv, dsgesv) as a function of the number of iterations $k$, where we can see how the performance varies with the increases of $k$. Usually, a small number of iterations is advantageous and can bring the highest performance, while a large number of iterations affects performance.

\section{NUMERICAL BEHAVIOR DISCUSSION}

Our experiments were performed on a system with two 10-core Intel(R) Xeon(R) E5-2650 v3 CPUs (20 cores total) running at $2.30 \mathrm{GHz}$ and one NVIDIA V100 PCIe GPU. To study the proposed methods and to highlight their practical use, we performed a large set of experiments on 21 types of matrices, with each type featuring different properties that represent a wide range of problems. We found that we could classify the 21 types of matrices using 6 representative cases. We first study the numerical behavior of our iterative refinement algorithms (e.g., dsgesv, dhgesv, and dhgesv-TC) each using either the IR or the IRGM solver, and show the convergence history of each technique for the different types of matrices.

This study aims to provide an analysis of each method's sensitivity relative to the matrix type as well as to provide insight into the performance expected from the iterative refinement methods. For example, if an iterative refinement method requires a large number of iterations to achieve FP 64 solution accuracy for a certain matrix type, then we can expect that its performance will degrade relative to the standard dgesv and it may be even slower. We note that the number of iterations that we report is the total number of iterations-including the inner GMRES iterations in the case of the IRGM solver. This means that, in both cases, the number of iterations is a precise indicator of the time spent in the refinement loop.

Table [I] describes the different matrix types and sizes used in our experiments.

TABLE II

DESCRIPTION OF THE TEST MATRICES, WHERE COND IS $\kappa(A)$.

\begin{tabular}{|l|l|l|l|}
\hline Type & \multicolumn{3}{|c|}{ Description } \\
\hline 1 & - & Random numbers with diagonal modified to be dominant \\
\hline 2 & $\lambda>0$ & Random $\sigma$ in $\left[\frac{1}{\text { cond }}, 1\right]$ such that their logarithms are uniformly \\
\hline 3 & $\lambda>0$ & Clustered $\sigma$ & $\sigma=\left[1, \cdots, 1, \frac{1}{\text { cond }}\right]$ \\
4 & - & Clustered $\sigma$ & $\sigma=\left[1, \cdots, 1, \frac{1}{\text { cond }}\right]$ \\
\hline 5 & $\lambda>0$ & Arithmetically distributed $\sigma$ & $\sigma_{i}=1-\left(\frac{i-1}{n-1}\right)\left(1-\frac{1}{\text { cond }}\right), i=1 . . n$ \\
6 & - & Arithmetically distributed $\sigma$ & $\sigma_{i}=1-\left(\frac{i-1}{n-1}\right)\left(1-\frac{1}{\text { cond }}\right), i=1 . . n$ \\
\hline
\end{tabular}

Figure 5 show the convergence history of the six proposed solvers (the three precision implementations each using either IR or IRGM). They are labeled as FPXX $\rightarrow$ FP 64 YY, where " $X X$ " corresponds to the algorithm used for the LU factorization (FP16-TC, FP16, or FP32), and "YY" represents the iterative refinement solver (IR or IRGM) used to attain FP 64 solution accuracy. In Figure 5a, we display the most numerically favorable type of matrix to solve- the diagonally 


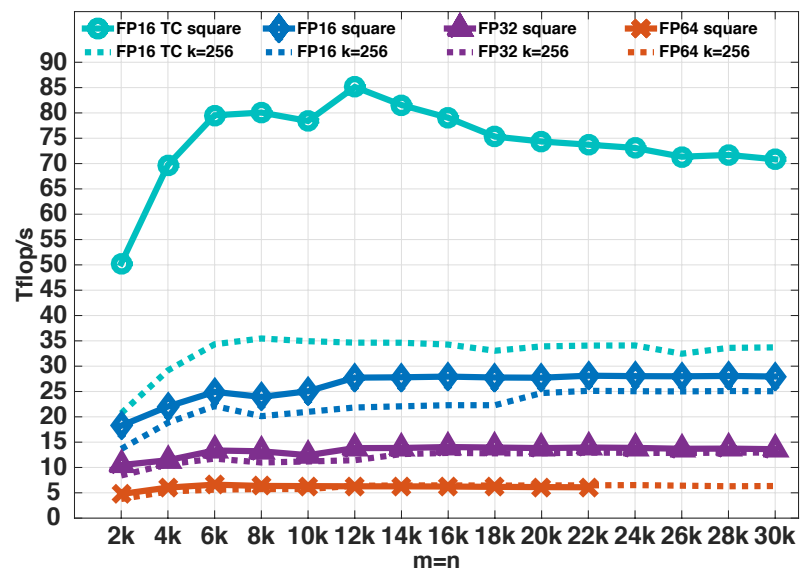

(a) Performance of the Xgemm used in the Xgetrf rank-k updates.

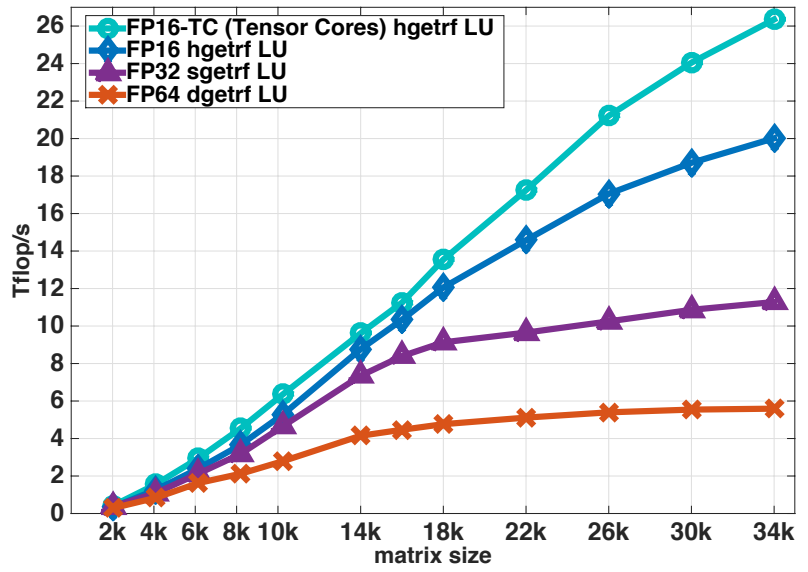

(b) Performance of the Xgetrf routine.

Fig. 3. Performance of the three arithmetic precisions obtained on a Nvidia V100 GPU.

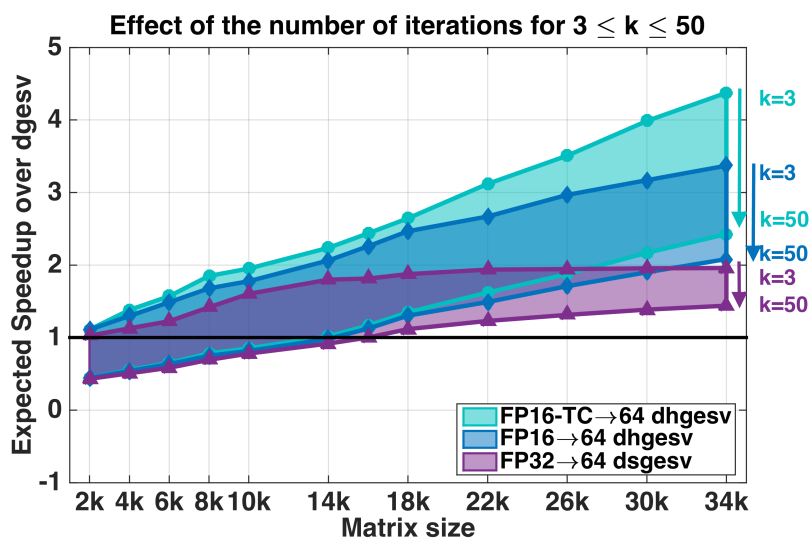

Fig. 4. Expected speedup of the three MP routines over the dgesv routine as function of the number of iterations and the matrix size.

dominant matrix. Here, we can see that all six variants converge in 3-5 iterations. For this type of matrix, since the number of iterations is small we can expect a large speedup over the FP 64 routine.

We believe that the FP 32 routine will achieve a $2 \times$ speedup and that both of the EP16 routines will achieve about $3 \times-$ $4 \times$ speedup while delivering a solution at FP 64 accuracy. More details about the performance are provided in the next section. Figure $5 b$ represents a matrix type that has positive eigenvalues and singular values for which the logarithms are uniformly distributed between 1 and $\frac{1}{\text { cond }}$. This is slightly more difficult than the diagonally dominant type. We observe that the convergence of the FP32 remains stable at 3 iterations, while the FP16 slightly increases to about 7-8 iterations. Interestingly, the FP16-TC converges faster than the FP16 and slightly slower than the FP32. This is because the accumulation in the FP16-TC rank-k update is in FP32 arithmetic and thus produces a better result than the FP16. This behavior can be seen on all graphs in Figure 5.

Figure $5 \mathrm{c}$ shows a more difficult type of matrix with clustered singular values and a positive eigenvalue. The FP32 method using either IR or IRGM converges as expected in 3 iterations. The FP16 IR variant converges very slowly and needs more than 400 iterations to drive the solution to $10^{-9}$ accuracy. However, the IRGM solver (FP16 IRGM) achieves the FP 64 solution accuracy in about 14 iterations. This reveals the sensitivity of the FP16 IR variant and highlights the importance of using the preconditioned GMRES solver inside the iterative refinement process. We note that GMRES delivers a more stable solution to $A c=r$ inside the refinement process, which allows the IRGM method to converge faster than IR. We also note that the diamond marker in the blue dashed linethe curve that represents FP16 IRGM-shows the number of outer iterations (refinements) in the IRGM solver. We can see that the number of outer iterations is about 4 , which also proves the theory that the solution of $A c=r$ delivered by GMRES is good enough to make the refinement loop converge in 4 iterations. More details about using GMRES inside the refinement process can be found in [4]. The EP16-TC variants using either IR or IRGM converge in 4 iterations. This underlines the importance of the FP 32 accumulation done in the hgemm-TC routine used in the FP16-TC variant. The FP16-TC variant works well for this type of matrix, and we can expect about a $4 \times$ speedup.

Figure $5 \mathrm{~d}$ shows results on matrices with the same singular value distribution as in Figure 5c but with complex eigenvalues and of different positive and negative sign. When comparing the two figures, we notice the effect of having positive eigenvalues. We also note that the FP 32 routine is not affected and converges in 4 iterations. The convergence of FP16-TC decreases slightly, requiring $\approx 13$ iterations. The FP16 IR variant diverges, which highlights the problem with using trsv in the classical iterative refinement to compute the correction, as it becomes unstable when the LU factorization is performed in FP16 and cannot bring the solution down to an acceptable accuracy. While using GMRES, the FP16 IRGM can bring the solution down to the FP 64 accuracy in $\approx 63$ iterations. 

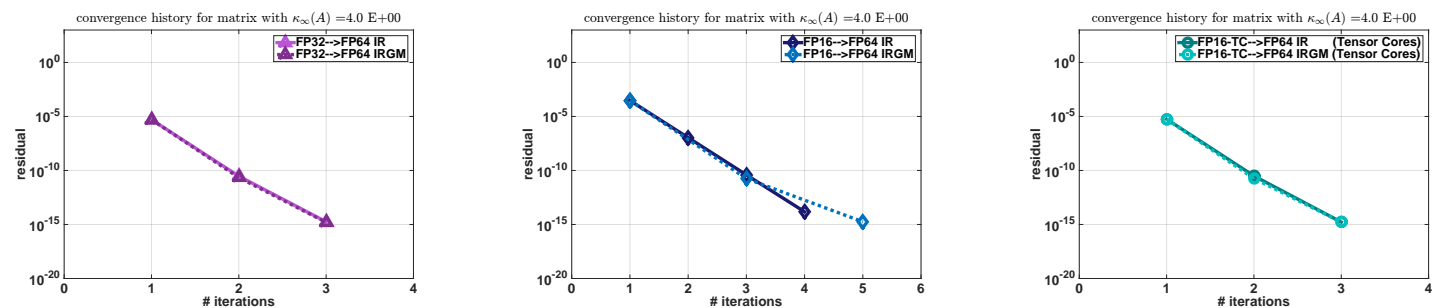

(a) Matrix of type 1: diagonally dominant.
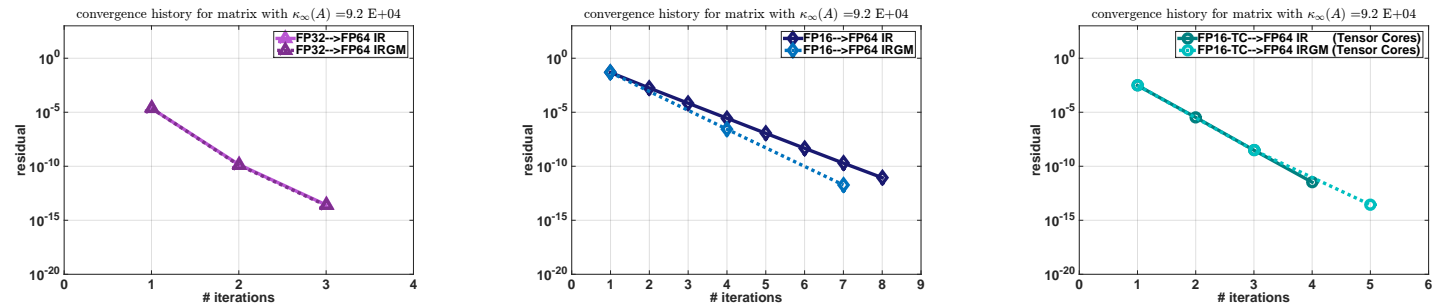

(b) Matrix of type 2: positive $\lambda$, where $\sigma_{i}$ is a random number between $\frac{1}{\text { cond }}$, and 1 such that their logarithms are uniformly distributed.
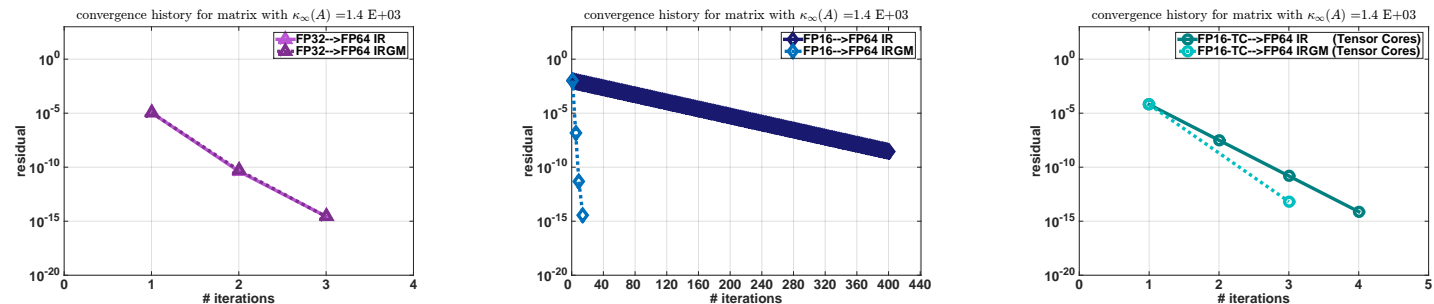

(c) Matrix of type 3: positive $\lambda$ with clustered singular values, $\sigma_{i}=\left(1, \cdots, 1, \frac{1}{\text { cond }}\right)$.
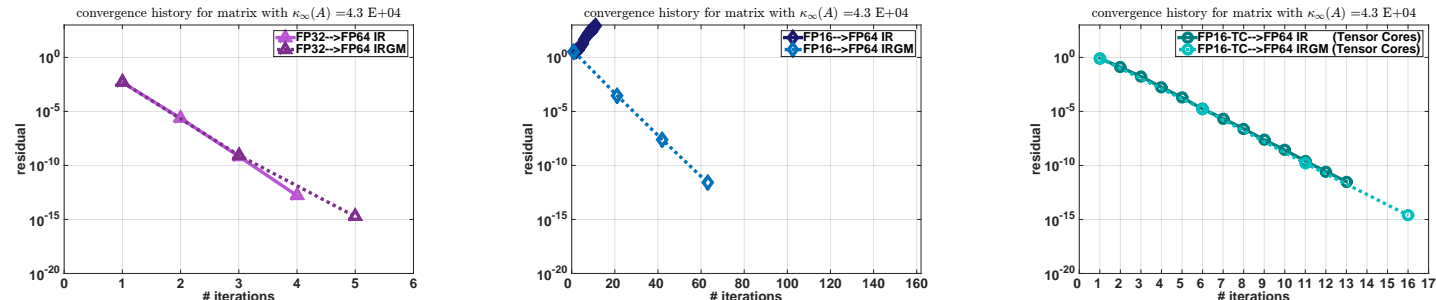

(d) Matrix of type 4: clustered singular values, $\sigma_{i}=\left(1, \cdots, 1, \frac{1}{\text { cond }}\right)$.
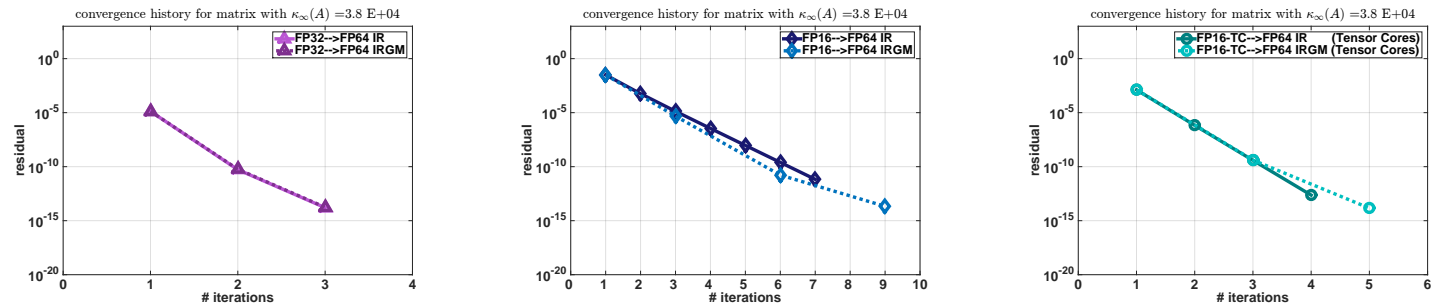

(e) Matrix of type 5: positive eigenvalues and arithmetic distribution of its singular values $\sigma_{i}=1-\left(\frac{i-1}{n-1}\right)\left(1-\frac{1}{\operatorname{cond}}\right)$.
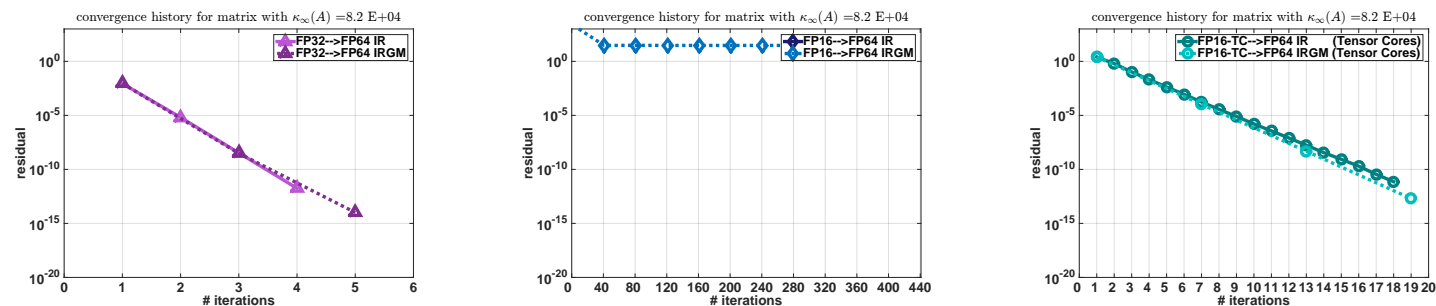

(f) Matrix of type 6: arithmetic distribution of its singular values $\sigma_{i}=1-\left(\frac{i-1}{n-1}\right)\left(1-\frac{1}{\text { cond }}\right)$.

Fig. 5. Convergence history of the two proposed iterative refinement algorithms (IR and IRGM) for the three precisions studied (FP16-TC, FP16, and FP32) and for different types of matrices-all of size $22,000 \times 22,000$, and having $\kappa_{\infty}(A)$ varying between $10^{1}$ and $10^{5}$. 
Figure 5e shows results on matrices where all methods behave well. Convergence was achieved in 3 iterations for FP 32, 4 iterations for EP16-TC, and 7 iterations for FP16. Figure $5 \mathrm{f}$ has the same singular value distribution as Figure $5 \mathrm{e}$ but not necessarily positive eigenvalues. This type is the most difficult, and the FP16 variants using either IR or IRGM do not converge. Note that the EP16 IRGM can converge when allowing more than 2,000 iterations, but for our experiment we limited the max iterations to 400 , since we have seen a large performance drop when iterations are around 200-where the iterative refinement becomes a drawback. The FP32 variants are not influenced by the matrix type and always converge in about 3-4 iterations. In contrast to the behavior for FP16, IR and IRGM converge in about 18 iterations for FP16-TC.

Lesson: For the matrices considered, the FP16-TC variant is the most robust and fastest in convergence of the two FP16 methods. The FP32 refinement variants show a consistent behavior regardless of the matrix types. This observation suggests the surprising effectiveness of the FP16-TC arithmetic, which might be robust enough to be used in HPC dense linear system solvers.

\section{EXPERIMENTAL RESULTS DISCUSSION}

This section presents the performance results of our three iterative refinement methods-dhgesv-TC, dhgesv, and dsgesv-using IRGM, compared to the reference dgesv solver. We also depict the number of iterations required by each method to reach EP64 accuracy. The teraFLOP/s are computed based on the same formula $\left(P=\frac{2 n^{3}}{3 \text { time }}\right)$, which means performance reflects the time to solution (e.g., if a method has $2 \times$ higher performance, it is $2 \times$ faster). The performance results are presented in Figures 6 and 7 for the six representative types of matrices studied in Section VI. In each figure, there are four performance curves that refer to the reference dgesv and to the three iterative refinement algorithms, dhgesv-TC, dhgesv, and dsgesv.

In Figure 6a, the matrix is diagonally dominant, andas shown in Section VI-all variants require three to four iterations to converge. Thus, one can expect that the low precision iterative refinement algorithms will bring a large speedup compared to dgesv. Since the number of iterations is small, we imagine that the speedup ratio will be similar to the one observed in Figure $3 b$ for the LU factorization. We confirm our expectation by looking at Figure 6a. The FP16-TC dhgesv-TC routine delivers a solution $4 \times$ faster than its FP 64 dgesv counterpart. Similarly, the dhgesv variant shows $a \approx 3 \times$ speedup over the dgesv, and the dsgesv variant shows $a \approx 1.8 \times$ speedup over the dgesv. This is example illustrates the importance of using the low EP16-TC precision in HPC.

Figure $6 \mathrm{~b}$ shows the performance of our methods for matrices with positive eigenvalue and logarithmic uniform distribution of their singular values. As shown in the figure, and similar to the previous graph in Figure $6 a$, the number of iterations remains constant when the matrix size increases for all algorithms. This type of matrix is marginally more difficult for the $\mathrm{FP} 16$ variant than the diagonally dominant matrix type-about 7 iterations vs. 3 iterations (Figure 6a). The FP16-TC and EP 32 variants require two to three iterations for both examples. Thus, one can expect the performance gain to be roughly similar to the diagonal dominant example. The dhgesv-TC (FP16 $\rightarrow$ FP 64) variant results in a speedup of $4 \times$ over dgesv. The dhgesv (FP16 $\rightarrow$ FP 64) achieves the same solution as the dgesv and is about $3 \times$ faster, while the dsgesv (FP32 $\rightarrow$ FP 64) is about $1.7 \times$ faster.

Figure $7 \mathrm{a}$ supports our findings that low precision techniques can be used to speedup a linear solver by a large factor. The performance results depicted here are similar to the previous two examples, where dhgesv-TC, dhgesv, and dsgesv outperform dgesv and provide around $4 \times, 2.6 \times$ and $1.7 \times$ speedups, respectively. In contrast to Figure $7 \mathrm{a}$. Figure $7 \mathrm{~b}$ shows the performance and the number of iterations for matrices that have similar clustered singular value distribution, but their eigenvalues are not necessarily positive and can be even complex. The observation made here is interesting. The behavior of the dsgesv (FP32 $\rightarrow$ FP 64) variant remains the same as in the previous experiments, requiring two to three iterations independent of the matrix size or matrix type. Thus, we will always see a $1.7 \times$ speedup. For the dhgesv-TC, the number of iterations increases compared to the previous examples (10-14 iterations vs. 3 iterations). We also see that the iteration count increases slightly with matrix size. Thus, one can expect the performance of the dhgesv-TC here to be slightly lower than in the previous example (while still being about $3 \times$ faster).

For the dhgesv, the number of iterations increases dramatically with the matrix size and is larger than what was depicted in Figure $7 \mathrm{a}$ In this case, the rounding error of the EP16 method-and possibly the range of the representative numbers for FP 16 arithmetic_-disturb the LU factorization. As a result, the convergence rate decreases dramatically, which then affects the performance. In this case, we can see that dhgesv is not beneficial at all and can be slower than FP 64. For such matrix types, the best practice is to use either the Tensor Core version dhgesv-TC, which provides a $3 \times$ speedup, or to use dsgesv, which yields a $1.7 \times$ speedup.

Figure 7c shows results for matrices with positive eigenvalues and an arithmetic distribution of their singular values. The dsgesv behavior stays the same as the one shown in the previous graph and requires about 2 iterations, resulting in a $1.7 \times$ speedup over dgesv. We also note that the dhgesv-TC routine acts similarly to dsgesv and converges in about 3 iterations, thereby making it an attractive routine to use in such cases, where it offers a speedup of around $4 \times$. The dhgesv behavior is comparable to the other problem types with positive eigenvalues, requiring about 7 iterations, regardless of the matrix size. Thus, we obtain a speedup of around $3 \times$. The results in Figure $7 \mathrm{~d}$ are similar to those in Figure $7 \mathrm{c}$ but without the constraint of positive eigenvalues. Here, we note that dsgesv still converges in about 3-4 iterations for any matrix size, leading to the observed $1.7 \times$ speedup, while the dhgesv fails to converge within 400 iterations for most of 


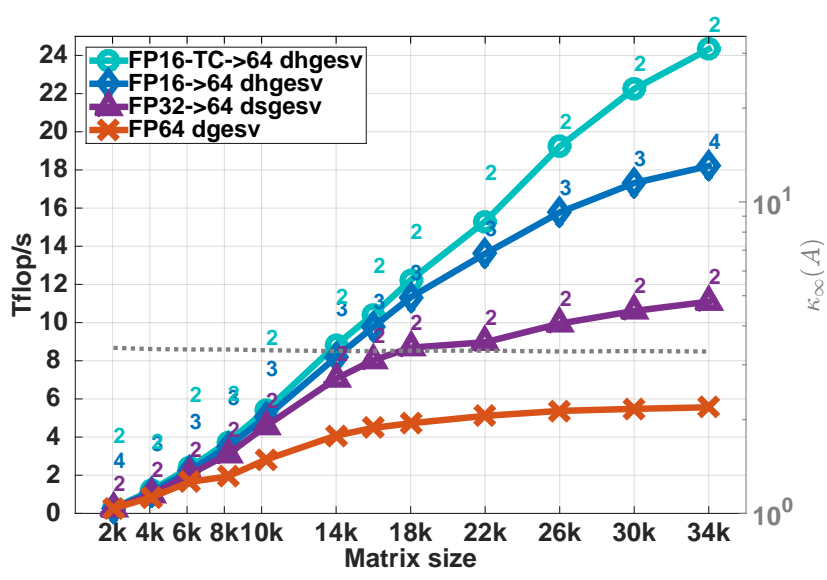

(a) Matrix of type 1: diagonally dominant.

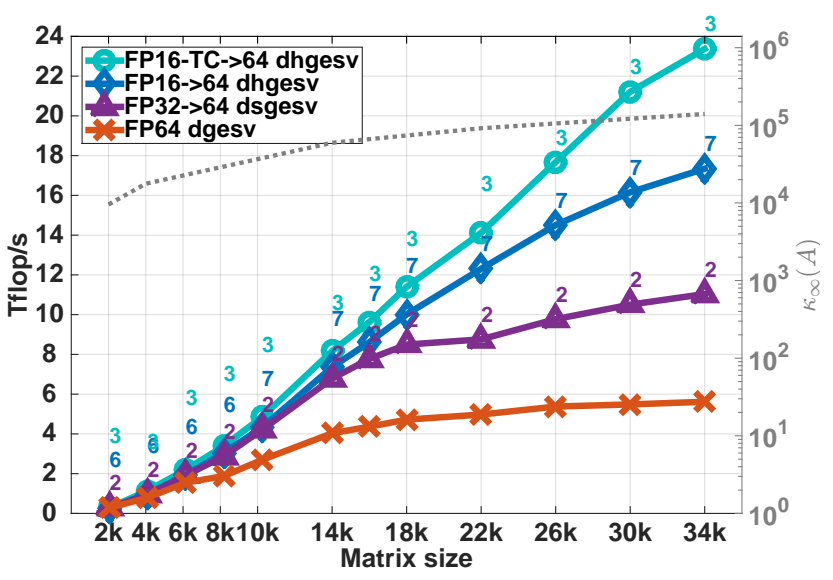

(b) Matrix of type 2: positive $\lambda$, where $\sigma_{i}$ is random number between $\frac{1}{\text { cond }}$ and 1 is such that their logarithms are uniformly distributed.

Fig. 6. Performance in teraFLOP/s of the four proposed linear solvers (one standard solver and three iterative refinement solvers, respectively) for different matrix sizes and different matrix types: 1) the FP 64 standard dgesv solver (orange color with " $\times$ "), 2) the FP 32 solver dsgesv (purple color with " $\triangle$ "), 3) the FP16 solver dhgesv (blue color with " $\diamond$ "), and 4) the FP16-TC solver dhgesv-TC (cyan color with "o"). For the iterative refinement solvers, we also depict the required number of iterations to achieve the FP 64 arithmetic solution, and we note that "nc" mean no convergence after 200 iterations. Note that the right " $y$ " axis shows the condition number, $\kappa_{\infty}(A)$, corresponding to the gray dotted line.

the large matrices, thereby making dhgesv useless in this case and validating our discussion of Figure $5 \mathrm{f}$ in Section $\mathrm{VI}$ The attractive revelation is the FP16-TC implementation. The V100 GPU's FP16-TC feature does accumulation in FP32 arithmetic and is able to fix the issue of the FP16; in doing so, it converges in about 10-20 iterations, netting a speedup of around $3 \times$. The goal of this paper is to show that the proposed IR solver can be of great benefit for a wide range of matrices with different characteristics. In practice, the real world matrices tend to be easier to deal with than our specially constructed ones. To illustrate this, we show results for real world matrices arising from different problems. We show in Table III the results from experiments obtained when running the four proposed linear solvers (one standard solver and three iterative refinement solvers, respectively) for different real world matrices from the SuiteSparse Matrix Collection (previously called the University of Florida Sparse Matrix Collection) [6] and dense matrices arising from electromagnetism radar design. As can be seen, the behavior of the proposed IR solver is similar to that for the synthetics matrices. The dhgesv-TC routine can provide about $4 \times$ speedup for a wide range of real world matrices while the dhgesv brings around $3 \times$ speedup and the dsgesv show about $1.7 \times$ speedup.

Lesson: The speedups presented in Figures 6 and 7 confirm our numerical analysis from Section VI, where we noted that iterative refinement algorithms are advantageous and exhibit very good speedups. The dhgesv-TC (FP16-TC $\rightarrow$ FP 64) routine can be used for all matrix types and for any matrix size to provide speedups of about $3 \times-$ $4 \times$, and the dsgesv (FP32 $\rightarrow$ FP 64) routine can be used for all matrix types and for any matrix size to provide speedups of about $1.7 \times$. The number of iterations is constant for all matrix types and matrix sizes for dsgesv.
It is also constant for dhgesv-TC for matrices with positive eigenvalues and only slightly increases for other cases. The dhgesv routine (FP16 $\rightarrow$ FP 64) is acceptable and provides a speedup of around $2.6 \times$ when the matrix has good properties (e.g., diagonal dominance) or when eigenvalues are always positive.

\section{Conclusions And Future Directions}

We have developed a framework of algorithms and their high-performance implementations for exploiting GPU Tensor Cores in mixed-precision FP16-FP32/FP64 iterative refinement solvers. We demonstrated for the first time how to use the Tensor Core to provide an additional FP16-TC performance boost to solvers in high FP64 accuracy. We provided results and analysis for a number of algorithms on different types of matrices. Specifically, we showed practical cases where even a highly optimized FP 64-precision solver, running at 6 teraFLOP/s, can be accelerated up to $4 \times$. The new developments introduce a new class of mixed-precision dense matrix factorization algorithms that can be used as building blocks in other mixed-precision algorithms.

The developments open up opportunities for future work directions, including further optimizations, development of a full set of mixed-precision factorization routines, and release as open-source software through MAGMA [15]. We also would like to mention that the aim of this paper is to show that mixed precision techniques are of great interest for linear solver in many engineering areas. Such methods can be easily ported to distributed or multi-GPU environments. Based on our record of track developing distributed libraries, we believe, that the speedup of the lower precision versus the double precision solver observed for these experiment on one node will remain the same for distributed environment because it 


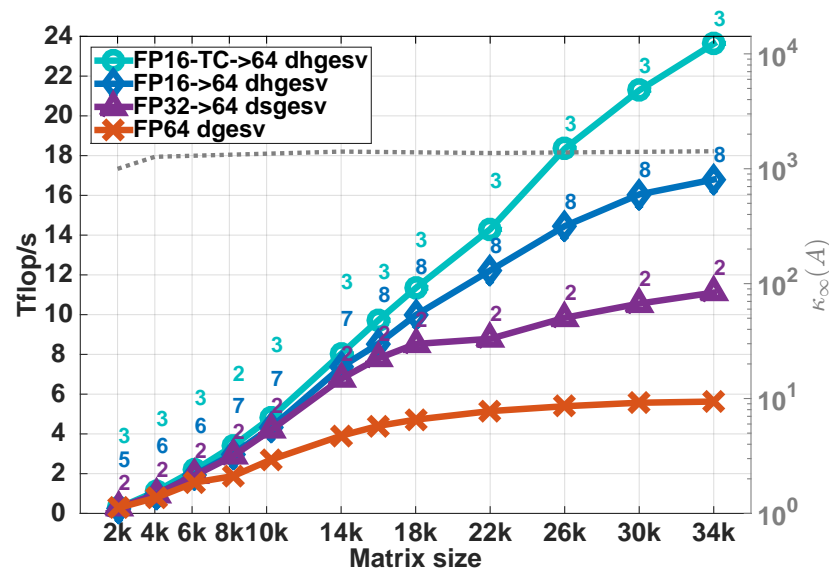

(a) Matrix of type 3: positive $\lambda$ with clustered singular values, $\sigma_{i}=(1, \cdots, 1$, $\frac{1}{\text { cond }}$.

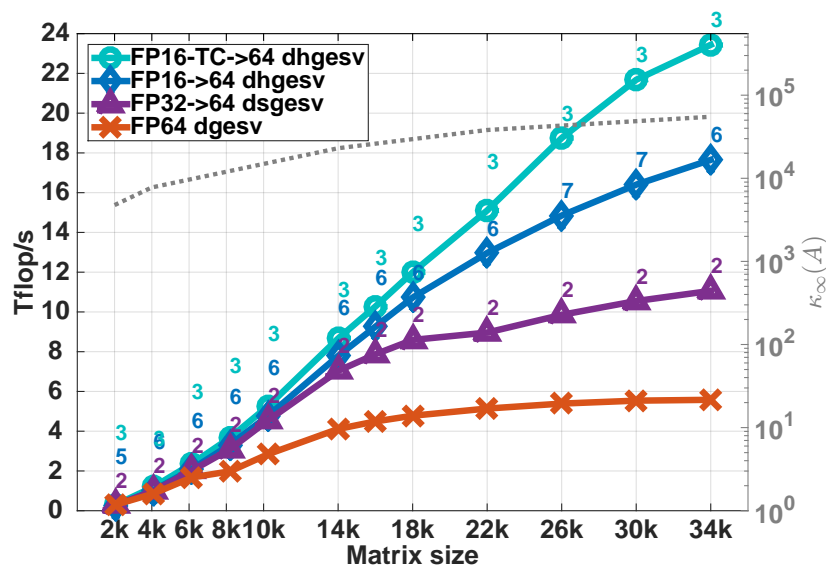

(c) Matrix of type 5: positive eigenvalues and arithmetic distribution of its singular values, $\sigma_{i}=1-\left(\frac{i-1}{n-1}\right)\left(1-\frac{1}{\text { cond }}\right)$.

Fig. 7. Performance in teraFLOP/s of the four proposed linear solvers (one standard solver and three iterative refinement solvers, respectively) for different matrix sizes and matrix types: 1) the FP64 standard dgesv solver (orange color with " $\times$ "), 2) the FP32 solver dsgesv (purple color with " $\triangle$ "), 3 ) the FP16 solver dhgesv (blue color with " $\diamond$ "), and 4) the FP16-TC solver dhgesv-TC (cyan color with "o"). For the iterative refinement solvers, we also depict the required number of iterations to achieve the FP 64 arithmetic solution, and we note that "nc" mean no convergence after 200 iterations. Note that the right " $y$ " axis shows the condition number, $\kappa_{\infty}(A)$.

TABLE III

PERFormance For REAL-LIFE MATRICES From the SUITESPARSE COllection [6] AND FROM DENSE MATRIX ARISING FROM RADAR DESIGN

\begin{tabular}{|c|c|c|c|c|c|c|c|c|c|c|}
\hline \multirow[t]{2}{*}{ name } & \multirow[t]{2}{*}{ Description } & \multirow[t]{2}{*}{ size } & \multirow[t]{2}{*}{$\kappa_{\infty}(A)$} & \multirow{2}{*}{$\begin{array}{l}\text { dgesv } \\
\text { time(s) }\end{array}$} & \multicolumn{2}{|c|}{ dsgesv } & \multicolumn{2}{|c|}{ dhgesv } & \multicolumn{2}{|c|}{ dhgesv-TC } \\
\hline & & & & & \# iter & time $(\mathrm{s})$ & \# iter & time $(\mathrm{s})$ & \# iter & time $(\mathrm{s})$ \\
\hline em192 & radar design & 26896 & $10^{6}$ & 5.70 & 3 & 3.11 & 40 & 5.21 & 10 & 2.05 \\
\hline appu & NASA app benchmark & 14000 & $10^{4}$ & 0.43 & 2 & 0.27 & 7 & 0.24 & 4 & 0.19 \\
\hline $\mathrm{ns} 3 \mathrm{Da}$ & 3D Navier Stokes & 20414 & $7.610^{3}$ & 1.12 & 2 & 0.69 & 6 & 0.54 & 4 & 0.43 \\
\hline nd6k & ND problem set & 18000 & $3.510^{2}$ & 0.81 & 2 & 0.45 & 4 & 0.36 & 3 & 0.30 \\
\hline $\mathrm{nd} 12 \mathrm{k}$ & ND problem set & 36000 & $4.310^{2}$ & 5.36 & 2 & 2.75 & 3 & 1.76 & 3 & 1.31 \\
\hline
\end{tabular}

is mostly related to the speed of the operations (e.g., lower versus double) and because most of the optimized distributed LU solver hides the communication by what we call lookahead techniques. For that, we emphasize to observe the same trend of speedup in distributed and multi-GPU environment.Also of interest is investigating the energy efficiency of the approach compared to working precision implementations and other

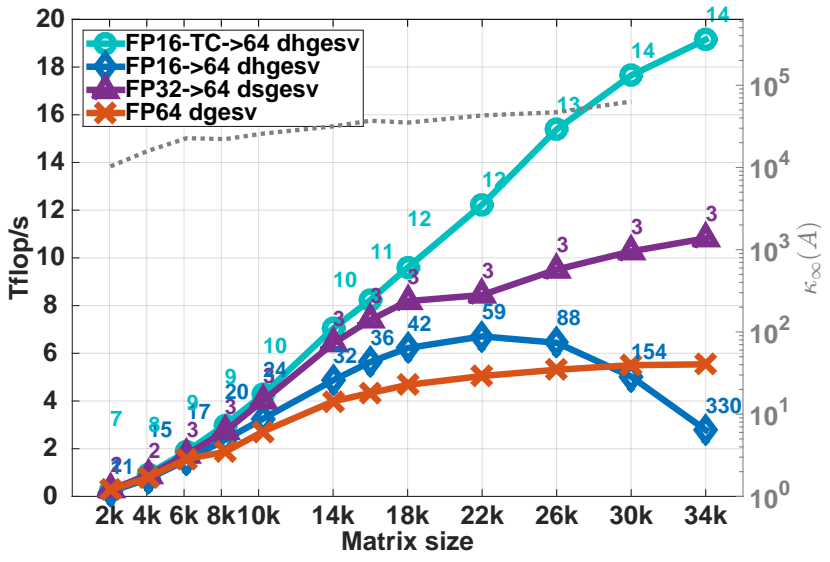

(b) Matrix of type 4: clustered singular values, $\sigma_{i}=\left(1, \cdots, 1, \frac{1}{\text { cond }}\right)$.

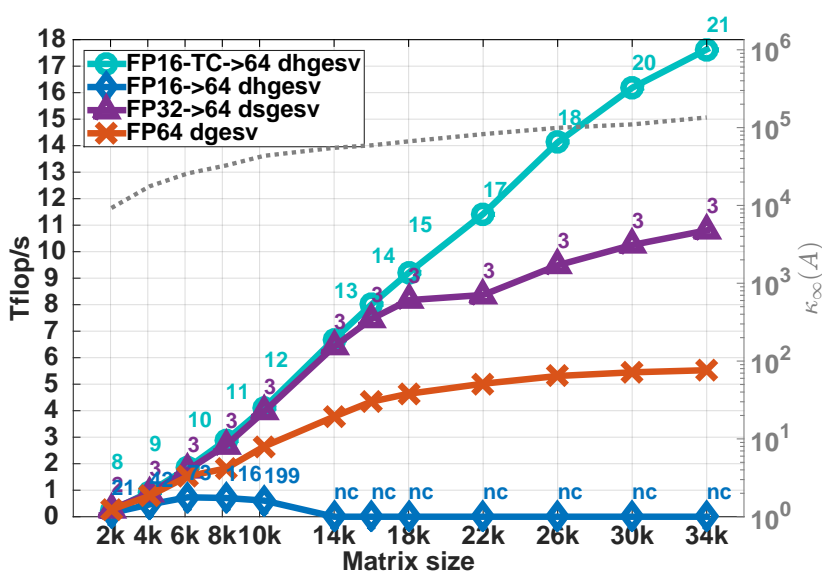
(d) Matrix of type 6: arithmetic distribution of its singular values, $\sigma_{i}=1-$ 


\section{ACKNOWLEDGMENTS}

This research was supported by the Exascale Computing Project (17-SC-20-SC), a collaborative effort of the U.S. Department of Energy Office of Science and the National Nuclear Security Administration. The work was also partially supported by Nvidia and NSF grant No. OAC-1740250. N. J. Higham was supported by Engineering and Physical Sciences Research Council grant EP/P020720/1, The MathWorks, and the Royal Society.

\section{REFERENCES}

[1] M. Arioli, J. W. Demmel, and I. S. Duff. Solving sparse linear systems with sparse backward error. SIAM J. Matrix Anal. Appl., 10(2):165-190, 1989.

[2] M. Baboulin, A. Buttari, J. Dongarra, J. Kurzak, J. Langou, J. Langou, P. Luszczek, and S. Tomov. Accelerating scientific computations with mixed precision algorithms. Computer Physics Communications, 180(12):2526-2533, 2009.

[3] A. Buttari, J. Dongarra, J. Langou, J. Langou, P. Luszczek, and J. Kurzak. Mixed precision iterative refinement techniques for the solution of dense linear systems. Int. J. High Perform. Comput. Appl., 21(4):457-466, Nov. 2007.

[4] E. Carson and N. J. Higham. A new analysis of iterative refinement and its application to accurate solution of ill-conditioned sparse linear systems. SIAM J. Sci. Comput., 39(6):A2834-A2856, 2017.

[5] E. Carson and N. J. Higham. Accelerating the solution of linear systems by iterative refinement in three precisions. SIAM J. Sci. Comput., 40(2):A817-A847, 2018.

[6] T. A. Davis and Y. Hu. The University of Florida Sparse Matrix Collection. ACM Trans. Math. Softw., 38(1):1:1-1:25, 2011

[7] J. W. Demmel. Applied Numerical Linear Algebra. SIAM, 1997.

[8] J. Dongarra, V. Eijkhout, and P. Łuszczek. Recursive Approach in Sparse Matrix LU Factorization. Scientific Programming, 9(1):51-60, 2001.

[9] G. H. Golub and Q. Ye. Inexact preconditioned conjugate gradient method with inner-outer iteration. SIAM Journal on Scientific Computing, 21(4):1305-1320, 2000.

[10] A. Haidar, A. Abdelfattah, M. Zounon, P. Wu, S. Pranesh, S. Tomov, and J. Dongarra. The design of fast and energy-efficient linear solvers: On the potential of half-precision arithmetic and iterative refinement techniques. In Y. Shi, H. Fu, Y. Tian, V. V. Krzhizhanovskaya, M. H. Lees, J. Dongarra, and P. M. A. Sloot, editors, Computational Science - ICCS 2018, pages 586-600. Springer International Publishing, Cham, 2018.

[11] A. Haidar, P. Wu, S. Tomov, and J. Dongarra. Investigating Half Precision Arithmetic to Accelerate Dense Linear System Solvers. In SC16 ScalA17: 8th Workshop on Latest Advances in Scalable Algorithms for Large-Scale Systems, Denver, CO, 11/2017 2017. ACM, ACM.
[12] N. J. Higham. Iterative refinement enhances the stability of $Q R$ factorization methods for solving linear equations. BIT, 31:447-468, 1991.

[13] N. J. Higham. Iterative refinement for linear systems and LAPACK. IMA J. Numer. Anal., 17(4):495-509, 1997.

[14] N. J. Higham. Accuracy and Stability of Numerical Algorithms. Society for Industrial and Applied Mathematics, Philadelphia, PA, USA, second edition, 2002.

[15] Matrix algebra on GPU and multicore architectures (MAGMA), 2018. Available at http://icl.cs.utk.edu/magma/

[16] H. Jagode, A. YarKhan, A. Danalis, and J. Dongarra. Power management and event verification in papi. In A. Knüpfer, T. Hilbrich, C. Niethammer, J. Gracia, W. E. Nagel, and M. M. Resch, editors, Tools for High Performance Computing 2015, pages 41-51, Cham, 2016. Springer International Publishing.

[17] J. Langou, J. Langou, P. Luszczek, J. Kurzak, A. Buttari, and J. J. Dongarra. Exploiting the performance of 32 bit floating point arithmetic in obtaining 64 bit accuracy. In Proceedings of the 2006 ACM/IEEE Conference on Supercomputing, 2006.

[18] X. S. Li and J. W. Demmel. Making Sparse Gaussian Elimination Scalable by Static Pivoting. In Proceedings of the 1998 ACM/IEEE conference on Supercomputing, number c, pages 1-17, 1998.

[19] C. B. Moler. Iterative refinement in floating point. J. ACM, 14(2):316$321,1967$.

[20] NVIDIA Management Library (NVML), NVIDIA, 2018. https:// developer.nvidia.com/nvidia-management-library-nvml

[21] Y. Saad. A flexible inner-outer preconditioned GMRES algorithm. Technical Report 91-279, Department of CSE, University of Minnesota, Minneapolis, Minnesota, 1991.

[22] Y. Saad and M. H. Schultz. Gmres: A generalized minimal residual algorithm for solving nonsymmetric linear systems. SIAM J. Sci. and Stat. Comput., 7(3):856-869, 1986.

[23] V. Simoncini and D. B. Szyld. Flexible inner-outer Krylov subspace methods. SIAM J. Numer. Anal., 40(6):2219-2239, 2002.

[24] R. D. Skeel. Iterative Refinement Implies Numerical Stability for Gaussian Elimination. Mathematics of Computation, 35(151):817-832, 1980.

[25] G. W. Stewart. Introduction to Matrix Computations. Academic Press, 1973.

[26] S. Tomov, J. Dongarra, and M. Baboulin. Towards dense linear algebra for hybrid gpu accelerated manycore systems. Parallel Computing, 36(5):232 - 240, 2010. Parallel Matrix Algorithms and Applications.

[27] S. Tomov, R. Nath, H. Ltaief, and J. Dongarra. Dense linear algebra solvers for multicore with GPU accelerators. In Proc. of the IEEE IPDPS'10, pages 1-8, Atlanta, GA, April 19-23 2010.

[28] J. H. Wilkinson. Rounding Errors in Algebraic Processes. Prentice-Hall, 1963. 